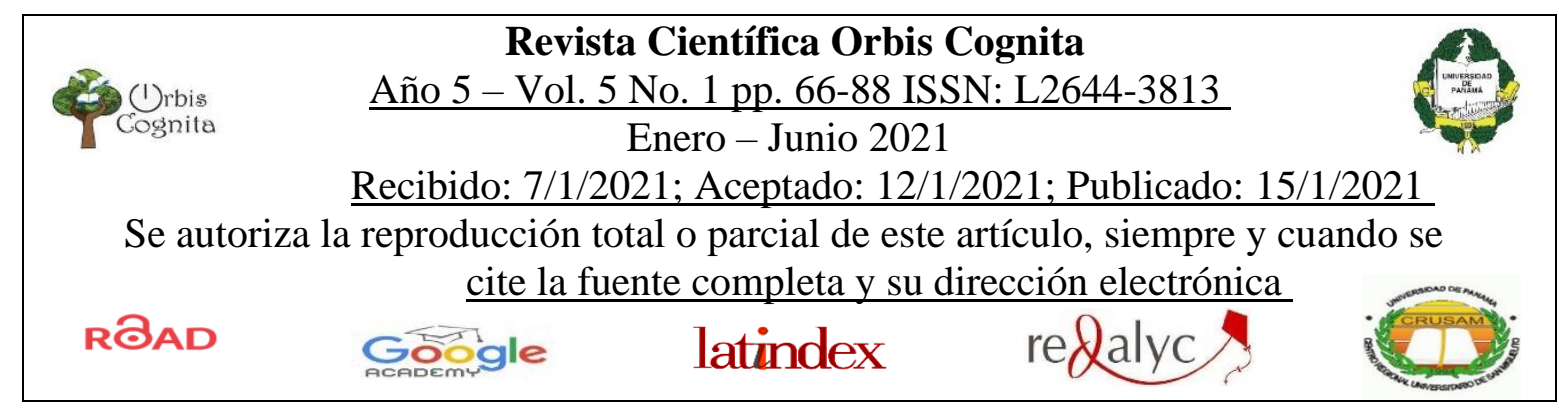

\title{
Complejidad y transdisciplinariedad: el desafío de los métodos centrados en la identidad
}

\section{Complexity and transdisciplinarity: the challenge of the methods focused on identity}

Karina A. Bustos, Vincy Consultora. Argentina

kbalunco@gmail.com 0RCID 0000-0002-4305-3634

Carlos J. Delgado, Universidad de La Habana

cdelgado2001@gmail.com ORCID 0000-0002-96054219

Giampiero Finocchiaro, Vincy Consultora. Italia

figipi@gmail.com ORCID 0000-0002-4578-5353

Melisa D. Godoy², Javier. A. Navarra², Magdalena Pérez Bruno ${ }^{2}$

2 Instituto de Docencia e Investigación de Fundación AlunCo, Argentina

lic.melisagodoy@gmail.com ORCID0000-0003-0504-7492

javiernavarra@gmail.com ORCID 0000-0002-4219-1197

mperezbruno@gmail.com ORCID 0000-0001-5613-3249

\section{RESUMEN}

La separación disciplinaria resultante del avance de las ciencias y los saberes ha generado sesgos respecto del contacto, la comprensión y la acción en la complejidad y los niveles de la realidad. La transdisciplinariedad, al convocar el examen de los problemas reales del mundo, precisa una elaboración metodológica de diálogo. El nivel epistemológico de la metodología general no basta para encauzar acciones de transformación, se requiere la elaboración de metodologías que tomen en consideración los presupuestos epistemológicos, los contextos específicos y pongan a dialogar actores y saberes involucrados. El Método VETAS ${ }^{\circledR}$ sistematiza una metodología dialógica transdisciplinaria, opera como método centrado en la identidad, y permite aplicaciones en situaciones complejas de salud, las organizaciones y la educación. 
PALABRAS CLAVE identidad, Método VETAS®, complejidad, transdisciplinariedad, educación inclusiva

\begin{abstract}
The disciplinary separation resulting from the advancement of science and knowledge has generated biases regarding contact, understanding and action in the complexity and levels of reality. Transdisciplinarity, when calling for an examination of the real problems of the world, requires a methodological elaboration of dialogue. The epistemological level of the general methodology is not enough to direct actions of transformation, it requires the elaboration of methodologies that take into account the epistemological assumptions, the specific contexts and put actors and knowledge involved to dialogue. VETAS ${ }^{\circledR}$ Method systematizes a transdisciplinary dialogic methodology, operates as a method focused on identity, and allows applications in complex health situations, organizations and education.
\end{abstract}

KEY WORDS identity, VETAS®, complexity, transdisciplinarity, inclusive education

\title{
INTRODUCCIÓN
}

La humanidad ha pasado por múltiples y profundas revoluciones del conocimiento, superación de antiguos límites. El avance del conocimiento y del desarrollo tecnológico, se mueven en un cambio nunca antes experimentado. Es innegable que estas innovaciones fueron generando un impacto en la sociedad, en muchos casos mejorando nuestra calidad de vida y solucionando múltiples problemas.

La modernidad europea inició un proceso de diversificación de áreas de conocimiento que se fueron diferenciando cada vez más, a partir de sucesivas revoluciones científicas, lo que consolidó estructuras de investigación empírica y presupuestos teóricos generalizadores. Durante ese proceso, que tuvo sus orígenes en la antigüedad con la aparición de varios campos, la historia y la geometría entre ellos, se fueron delineando modos de investigación a partir de las correlaciones que se establecían con respecto a los objetos investigados. A ello, se añadieron 
los procesos de transformación social en las metrópolis, que contribuyeron a la creación de instituciones ocupadas de la ciencia y los conocimientos científicos, así como impulsos sociales y económicos a las actividades científicas en general, y especialmente a aquellas vinculadas a la producción. A partir de la entrada en la era de las revoluciones industriales, cada nuevo descubrimiento disparó la necesidad de ir por más, generando un circuito de transformación vertiginosa que ha dejado un conglomerado de nuevos conocimientos.

El acúmulo exponencial de nuevos avances, combinado con la exigencia de profundizar en cada uno de ellos, y la elaboración de generalizaciones teóricas en campos relativamente separados, fue provocando la "necesidad" de consolidar la separación de los conocimientos en distintas áreas. Emerge de estos procesos sociales, institucionales y cognoscitivos el resultado práctico de la tabicación propia de los saberes disciplinares. Por un lado, fue un resultado progresivo enorme, puesto que permitía un mejor abordaje de las realidades en estudio, y por otro, un retroceso a largo plazo, por la pérdida de conocimiento con respecto a las totalidades. La separación disciplinaria aceleró la matriz de innovaciones y su aplicación, así como favoreció nuestra comprensión de la realidad operando con nociones "simplificadas". Cada una de ellas, centrada en la especificidad de lo que se estudia y los métodos con que se estudia, permitió la estructuración disciplinaria de los conocimientos: cada disciplina estructura los conocimientos en función de un reducido número de variables facilitando la construcción de modelos explicativos capaces de abordar por "aproximación” la realidad estudiada. En este modelo el elemento común a todas las áreas es el de recorte y control del objeto de estudio. En este procedimiento van de la mano la reducción y la precisión. Se sustrae lo estudiado del contexto, y se le estudia en forma aislada. Esto permite comprenderlo mejor con respecto a la propiedad o la relación causal que se estudia. Lo que se afirma es exacto en el sentido de que han sido excluidas las influencias de factores aleatorios, y se han establecido con precisión los 
elementos capaces de actuar en el desencadenamiento de una relación, o los que la regulan y conducen.

Indudablemente se gana información con respecto al detalle, a la vez que se pierde información con respecto al conjunto, a la totalidad. Esto genera la certeza con respecto a que al comprender en profundidad los objetos aislados se devela la interacción propia de los mismos. Pero la certeza se torna fantasía cuando se trata de sistemas de sistemas, estrechamente interrelacionados, donde existen diversidad de variables, y donde no es posible realizar predicciones precisas por la falta de información, pero también y fundamentalmente, por la dinámica compleja que caracteriza a estos sistemas.

De este modo, a pesar del vertiginoso acúmulo de avances, la segmentación generó nuevos problemas que no pueden ser resueltos en los marcos de un modelo de reducción y simplificación.

\section{Disciplina y transdisciplina}

Un sistema de sistemas, específico, es el que abarca la problemática de la complejidad humana.

Se trata de problemas que corresponden a fenómenos biológicos, epidemiológicos, sociales, culturales, espirituales en general (mitológicos, religiosos, de creencias...) de estilos de vida y de afrontamiento, organizacionales, entre otros.

Cada uno de los elementos es fundamental para el conocimiento del conjunto, pues las posibles relaciones de causalidad que nos permitirían intervenir para realizar determinadas transformaciones o cambios en la situación inicial, ni dependen exclusivamente de un factor o número pequeño de factores, ni son necesariamente simétricas o estructuradas en jerarquías rígidas. Ante la inviabilidad de algunas soluciones disciplinarias estrechas, estás se han venido modificando, ajustándose en busca de un vínculo con lo que aportan otros campos disciplinarios, lo que ha dado lugar a diferentes mixturas interdisciplinarias, que muchas veces han contribuido a la aparición de nuevas disciplinas. Y, no obstante, los resultados continúan 
dejando un vacío o pérdida de información, que reclama cada vez la ampliación en busca de precisiones y nuevos vínculos con otros campos.

Además, resulta inevitable incluir elementos que se encuentran fuera de la ciencia, en la vida cotidiana, las costumbres, los modos de vivir, los imaginarios sociales, todo lo cual es difícil de manejar y estructurar en parámetros medibles.

El resultado ha sido el desmembramiento o desintegración de la realidad, ubicada por partes en cada disciplina, la aspiración a la integración, y la imposibilidad de alcanzarla mediante el diálogo que involucra solo a las disciplinas. La complejidad reclama atención, pero constantemente escapa a la lógica estrictamente disciplinaria.

Por otra parte, desde la perspectiva epistemológica, la disciplinariedad nace en un contexto en que las concepciones sobre el conocimiento estaban centradas en el conocimiento propio de los humanos. Durante los siglos de construcción de la ciencia disciplinaria, la atención epistemológica se movió en varias direcciones. Tres de ellas son importantes para el análisis que realizamos:

La dirección hacia la diversidad de los conocimientos.

De la atención casi exclusiva en el conocimiento humano se ha movido la atención hacia una concepción más amplia del conocimiento. De una parte, se ha comprendido que el conocimiento no es un producto exclusivo de la especie humana. El conocimiento humano es diverso, pero la diversidad del conocimiento atañe también al contexto general de la vida. Los seres vivientes producen conocimientos. No deberíamos usar el singular con respecto al conocimiento. Sería más correcto hablar siempre de los conocimientos.

La dirección hacia el rigor de la ciencia y el conocimiento científico. Se prestó atención especial a los modos de construir el conocimiento científico, a sus lógicas y los caminos para establecer un rigor lingüístico, terminológico, conceptual y de 
métodos. Este camino, que cobró fuerza durante el siglo XX en la filosofía de la ciencia, estableció parámetros y límites en las pretensiones de rigor, objetividad, modos de asumir la relación cognoscitiva, que han permitido delimitar la ciencia de la no ciencia, y a la vez, reconocer la diversidad de la ciencia y su riqueza metodológica. Como resultado, no deberíamos usar en la actualidad el término ciencia sin comprometernos con determinados modos de hacer, históricamente determinados, pero específicos de ella, muy diferentes de los parámetros de validez de la vida cotidiana, y con especificidades considerables cuando nos movemos de una ciencia a otra (como ocurre, por ejemplo, entre "ciencias naturales", "ciencias sociales", y "humanidades"). Como resultado, el rigor de la ciencia se acompaña del reconocimiento de su diversidad.

La dirección hacia la evolución del conocimiento humano ha mostrado que la constitución de un sujeto humano incluye además de los elementos biológicos, sociales, culturales, e históricos, un devenir específico a nivel individual, que se va modificando en el transcurso del tiempo. Así, la epistemología evolutiva coloca atención en la diversidad específica del sujeto humano, lo que impacta la comprensión del sujeto científico desde otro ángulo: el de su pertenencia a un universo de cultura que se modula en el propio proceso de constitución biológica del sujeto. Desde entonces, comprendemos que la etapa evolutiva que la edad de cada individuo representa, impacta las producciones cognoscitivas que genera.

La disciplinariedad resulta entonces de un proceso doble, de configuración, estructuración y constitución de la ciencia centrada en ámbitos o áreas de estudio que se intenta abarcar a partir de procedimientos que han venido precisando y cambiando, proceso que se acompaña del cambio en las concepciones epistemológicas y los modos de entender los conocimientos. Así 
llegamos al punto en que la excelencia incuestionable de la ciencia disciplinaria choca con los límites que para ella representa la complejidad de lo investigado.

El desafío de la complejidad consiste en el reconocimiento de la necesidad de situar el objeto de estudio, de abordarlo, sin separarlo del tejido a que pertenece y le constituye. Esto significa que en una relación compleja lo que se estudia puede ser entendido como una identidad dinámica, es decir, identidad en cambio (que es, y para ser, no tiene otro camino que dejar de ser). Parte de esa identidad puede integrar formas diversas de conocimientos. En esos casos, de seres vivientes, seres humanos y dispositivos técnicos, la complejidad emergente incluye en el entretejido los conocimientos.

El conocimiento complejo intenta reconocer lo que vincula al objeto con su contexto, el proceso o la organización en la que se inscribe. En efecto, el conocimiento es más rico, más pertinente en cuanto se lo relaciona con hechos, elementos, procesos, informaciones, datos, reconocidos dentro del contexto donde se integran. Esto significa que el desafío de la complejidad exige la comunicación entre los conocimientos separados; demanda, al mismo tiempo, principios de organización del conocimiento que permitan vincular los saberes de manera pertinente, incluyendo también a la práctica profesional.

Cuando se trata del sujeto humano y el estudio de problemas de salud o educación, la complejidad se distingue por lo imbricado de ese tejido biológico, cognoscitivo y social. No es sólo la vertiginosidad del cambio actual lo que nos afecta, sino todo aquello que forma parte de nuestro sistema de valores, la inestabilidad permanente de lo aceptado como verdadero, los nuevos fenómenos socio-económicos y la transformación producto de nuestro mundo bajo influencia de los cambios científicos y tecnológicos.

Todo esto exige una nueva contextualización del sujeto y hace necesario reconsiderar su humanidad (incluidos los aspectos culturales, su diversidad social y sus sistemas de valores). No obstante, muchos de esos aspectos se han movido en dirección contraria, es decir, se han 
ido desdibujando en favor de las normativas que objetivan el conocimiento científico, ya sea en anclajes físicos, biológicos, bioquímicos, neurocientíficos. Estos anclajes ciertamente pueden aportar precisiones en cuanto a la realidad natural como la física, química y biológica de los sujetos, pero no agotan el sujeto y dan cuenta de manera muy limitada y distante, de la realidad social y cultural que les constituye. Para entender la salud, indudablemente las cuestiones fisicoquímicas y biológicas son relevantes, pero la salud no se reduce a ellas. Lo que queda fuera de la ecuación es relevante, pero queda omitido cuando se simplifica su complejidad. De manera semejante ocurre en educación y la amplia gama de fenómenos sociales.

La transdisciplinariedad se plantea como un enfoque epistemológico para intentar abarcar este tipo de situaciones complejas, donde no basta con reducir una realidad a otra. Al prestar atención al mismo tiempo a aquello que está entre, a través y más allá de las disciplinas, no se menosprecia el conocimiento disciplinario, sino que se le acompaña, se le complementa mediante el diálogo con lo que ha quedado fuera. No se trata de una anti disciplina, o de la negación del conocimiento disciplinario, sino de un diálogo crítico con la diversidad de los conocimientos que deben considerarse al estudiar determinada realidad. En el horizonte se reafirma la unidad del conocimiento con y más allá de las disciplinas.

La transdisciplinariedad genera una posible respuesta metodológica general a la problemática del reduccionismo planteada anteriormente (Nicolescu 2009, p. 13-38). Se pretende con ella armonizar lo que se encuentra fraccionado en disciplinas, integrar lo que de ellas proviene y es valioso para la comprensión de la realidad, entendida en niveles (niveles de realidad) que, en su resistencia a ser reducidos, reclaman la complementariedad y la integración como abordajes adecuados al entretejido que constituyen y al que pertenecen. Los problemas de estudio se reconocen como complejos, es decir, problemas propios de sistemas con más de un futuro, de manera que el diálogo de saberes debería contribuir a la delimitación de esos futuros posibles, 
y la probabilidad de su ocurrencia. Así la transdisciplinariedad se establece como principio de unidad de los conocimientos, más allá de las disciplinas involucradas, que cobra sentido siempre que podamos identificar la existencia de más de un nivel de realidad que concurre en el entretejido estudiado (Nicolescu, 2005, p.5-14).

Lo antes expuesto permite evitar el error de considerar la transdisciplinariedad como una "super disciplina" que todo lo puede. Por el contrario, nos brinda un nuevo encuadre epistemológico para poder formular los problemas como complejos y a partir de esta reformulación, estar en condiciones de establecer nuevas formas o caminos para solucionarlos. De esta manera, no se desechan las disciplinas y los conocimientos disciplinarios, sino que se evita que la delimitación disciplinar de los objetos cercene la realidad de los mismos. Por otra parte, la solución epistemológica general aporta un marco, pero no basta, requiere de implementaciones metodológicas que hagan posible las acciones cognoscitivas y de transformación mediante el dialogo. Este nivel metodológico requiere trabajo de investigación-transformación en cada realidad especifica.

\section{Transito del método en general al nivel de la metodología}

La transdisciplina no es una forma de clasificación de las investigaciones, sino un modo de organización, de producción de los conocimientos. Este modo de organizar los conocimientos, implica que no basta con la comprensión filosófica y epistemológica general que la fundamenta: trabajar problemas reales del mundo requiere de una elaboración metodológica específica.

Dicha metodología debe contar con herramientas concretas que permitan materializar el accionar sobre la realidad, preservando los postulados de la transdisciplinariedad.

Tanto la metodología como sus herramientas deben ajustarse y operar como sistema abierto (y no como receta) en la comprensión y la intervención sobre el sistema particular (Nicolescu, 
2005, p.7-9). Lo anterior explica, por una parte, la imposibilidad de que existan expertos en transdisciplina, y por otra, la necesidad de realizar ajustes metodológicos cada vez que se trabaje asuntos específicos.

Un aspecto fundamental de la generación de métodos en transdisciplinariedad es el eje que trasciende. Sobre la base epistemológica que ofrece Nicolescu (1996), el estudio de una amplia base bibliográfica disponible (Delgado 2012, 2018; Freire 1969; Hurtado 2015; Klein 2013. Nicolescu, 1996, 2005, 2019) y la experiencia desarrollada en el Instituto de Docencia e Investigación de Fundación AlunCo ${ }^{1}$ en los estudios realizados entre 1998 y 2020, se elaboró una metodología cualitativa base para la investigación-transformación transdisciplinaria. La misma ha sido implementada y transferida mediante actividades de intervención y educación de postgrado (transferencias metodológicas, diplomaturas, becarías, fellowship, preceptorship, jornadas, plan de desarrollo de profesionales de la salud, la educación y las organizaciones) desarrolladas por la Fundación AlunCo (entre 1998 y 2020) y Vinci Consultora (entre 2017 y 2020).

Las actividades de postgrado llevadas adelante por la Fundación AlunCo y Vinci se implementaron en diplomaturas referidas a salud y a educación en universidades de gestión privada de Buenos Aires, incluyendo modalidades presenciales y virtuales, habilitando la participación en esta última, de participación activa de profesionales tanto de Argentina como de países limítrofes a ella. Las becarías de formación en clínica y en investigación transdisciplinarias bajo Método VETAS ${ }^{\circledR}$ conllevan el desarrollo de competencias de aplicación del método de profesionales de dichos ámbitos del territorio argentino. Los preceptorship y fellowship siguen la misma modalidad con criterios de tiempo más acotados para una pronta aplicación en los ámbitos de influencia de los profesionales en formación. Los

\footnotetext{
${ }^{1}$ El desarrollo del Instituto de Docencia e Investigación de la Fundación AlunCo comenzó en 1998 con impacto profundo en ámbitos de la salud, la educación y la acción social en Buenos Aires y ampliándose al territorio argentino.
} 
planes de desarrollo para profesionales de la misma Fundación AlunCo, buscan no sólo el mejoramiento constante del propio sistema sino el desarrollo de liderazgos que impacten en otros ámbitos de participación. Las jornadas, actividades más acotadas en tiempo, persiguen lograr mayor grado de conciencia que habilite luego una pregunta personal con criterio de investigación-transformación que se plasme en lo colectivo. Por otro lado, las transferencias metodológicas, resultan procesos de mayor transformación de los sistemas, ya que implican un diagnóstico colectivo de los agentes del sistema con los consultores para identificar los canales de apertura, aprendizaje y adaptación del Método VETAS® a una organización específica que tiene sus propios valores, visión, cultura, recursos y agentes de actuación, su propia identidad. El método desarrollado durante estos años asume el reto que representa la identidad, y se expresa como método centrado en la identidad (MCI).

La pregunta por la identidad parte de los grandes cuestionamientos que se dan en el centro mismo de la historia del ser humano. En los incansables intentos por definir la identidad humana se evidencia el desafío de la complejidad y la imperiosa necesidad de un método que pueda respetar su naturaleza.

A medida que se avanzó en la información detallada resultado de la especialización del método científico, se perdió lo global, lo transdimensional para terminar por deshumanizar y desconocer por completo lo identitario. Por otro lado, el efecto que produce categorizar la identidad para estudiarla, y desde estos desvíos de la reducción a la parte la pretención de generar nuevos métodos.

La identidad humana es en esencia dinámica y cambiante, producto de las representaciones históricas de una persona y en co-construción permanente con el medio, en esta dimensión dialógica y de trama indisociable se manifiesta su verdadera expresión y complejidad. 
Es en este postulado de la centralidad de la identidad que toma relevancia el tránsito de la disciplina a la transdiciplina, y que empuja un cambio metodológico, puesto que no existen identidades de laboratorio. La identidad en el centro de un método propone la restitución y la reconstrucción de la integridad de la persona. Posibilitar la integración de aspectos que permiten al individuo seguir siendo el mismo, a pesar de las diferentes experiencias que lo transforman, "metamorfosearse a su propio fin".

Los métodos centrados en la identidad plantean el fundamento hacia una verdadera transformación, brindando herramientas donde explorar lo que fuimos y nos constituye, lo que estamos siendo, pero escencialmente lo que todavía desconocemos de nosotros mismos. En ese aporte único, irrepetible e irrenunciable radica la creación de la innovación necesaria para un nuevo tiempo. El tiempo de la Identidad, la diversidad, la inclusión.

Como plantea Morin es importante hacer referencia a la recursividad, a pensar la restitución de la identidad no únicamente desde el individuo, sino también desde la identidad de los participantes de este proceso ya que existe un diálogo constante entre estas identidades que se manifiestan en una relación compleja de autonomía-dependencia (Morin, 2000).

Estamos tan identificados con Otros en este proceso de dependencias más que de autonomía, que jamás se nos ocurre cuestionar aquello que con certeza creemos que somos.

La tarea del "despertar identidad" puede considerarse entonces como un proceso central para cualquier método que aspire a la centralidad del ser humano. No es fácil comprender que, en el centro del ámbito organizacional, educativo, o que aspire a la salud, hay un proceso identitario que lo atraviesa y esto, exactamente, nos impone nuevas formas de diálogo.

No podemos reducir la identidad a un nivel disciplinario. ¿Cómo podríamos dividir al artista de su arte? 
Brindar entonces desde un método la posibilidad del descubrimiento continuo y dinámico hacia la aceptación e integración de las experiencias, la memoria y las creencias que permiten al individuo crear la atmósfera hacia la restitucuión de su identidad, debería convertirse en la brújula que guia el camino.

Centrarse en la identidad permite trabajar en contextos donde se presentan problemáticas de salud, organizacionales y educativas. La metodología de referencia recibe el nombre de Método VETAS®. Nació de la necesidad de atender un contexto específico como la discapacidad, y al centrarse en la identidad se ha podido extender más allá de ella, con validez para el trabajo en organizaciones y la educación.

\section{RESULTADOS TRANSDISCIPLINARIOS}

El Método VETAS®es resultado de los presupuestos teórico-epistemológicos antes expuestos, contrastados con la observación de prácticas clínicas deshumanizadas, y la emergencia de necesidades específicas que manifiestan fenómenos complejos como el abordaje de la discapacidad, y el déficit planteado por una formación profesional reduccionista.

La discapacidad, entendida como asunto reducible a un campo, la salud, condujo a enfoques reduccionistas para su atención, puesto que quedan fuera de su horizonte un conjunto importante de realidades. En sentido teórico, la transdisciplina demanda que se identifiquen los niveles de realidad (resistencia objetiva) a través de los cuales se manifiesta. Reconocer que no puede reducirse el asunto a una realidad única, es el primer paso para elaborar y responder la pregunta por la metodología adecuada para las intervenciones.

El cambio de paradigma radica en este caso, en considerar que además de un problema de salud, estamos ante un problema social, no como añadido o derivado, sino como una de las realidades de manifestaciones del fenómeno. Significa, no basta con entender la discapacidad 
como asunto de salud, ni es reducible la discapacidad a un asunto de salud. El reconocimiento de niveles de realidad potencia la desacomodación que surge de quererla encuadrar en los sistemas de conocimiento previos. Este fue el punto de partida para la construcción del método VETAS ${ }^{\circledR}$ que organiza los conocimientos disciplinarios orientándolos hacia un lenguaje común, la armonización de procedimientos y técnicas de intervención y gestión, a través del trabajo en equipo, para la resolución de problemas complejos.

En su forma original se trata de un método compuesto por procesos sistémicos, y centrado en la identidad -en este caso del paciente en neurorrehabilitación-, que trabaja hacia un objetivo común articulado por el trabajo en equipo y capaz de ser auditado en el ejercicio clínico en neurorrehabilitación.

Se lleva a cabo a través de módulos que incluyen como componentes principales la evaluación, el tratamiento y la inclusión social del paciente. Este método nos ha permitido llevar a la acción los postulados teóricos de la transdisciplina. Durante casi 20 años de aplicación del método ha ido creciendo y transformándose, manteniendo siempre una actitud transdisciplinaria que nos permite conservar un rumbo, una orientación al atravesar las situaciones complejas sorteando la tentación del reduccionismo y la atomización de la complejidad en elementos aislados.

En el devenir de esta puesta en acción de la transdisciplinariedad en el contexto de la discapacidad no solo se desarrollaron las estrategias, y manuales de procedimientos y gestión de la clínica enfocados a los aspectos de la salud. Fue necesario desarrollar experiencias en las áreas de gestión educativa y social. La salud ha sido el primer ámbito de implementación, y de ella se ha trazado un camino en el ámbito de la educación y de la acción social.

En sintonía con organismos internacionales como la UNESCO, y proyectos educativos internacionales emergentes que reconocen la necesidad de entender la educación en clave de complejidad y transdisciplina (Morin, 1999; UNESCO, 2015; Mussallam, 2019), se reconoce en un sentido amplio y humanitario, la necesidad de relacionar escuela y contenidos 
conceptuales, la construcción identitaria de la ciudadanía y la legitimación de los derechos humanos, la implicancia de valores como la conciencia, la comprensión y la diversidad para el desarrollo del potencial humano y la igualdad de oportunidades. Esta base a la vez teórica y práctica es el núcleo original del método que le permite trascender las situaciones que pueden identificarse inicialmente en un ámbito, por ejemplo, la salud, y terminan abordándose multilateralmente como sociales, culturales, organizacionales, educativas.

Dentro del ámbito de acción social, sabemos que a principios del siglo XXI la percepción de incertidumbre, indefensión e inseguridad es notoria en un gran porcentaje de la población latinoamericana. Estas percepciones se relacionan a condiciones de vida que se han visto acentuadas en términos del acceso al empleo, educación, ingresos, consumo, vivienda, crédito y seguridad social en el contexto de un nuevo patrón económico de desarrollo que ha emergido y consolidado en las últimas décadas.

La relevancia de la noción de vulnerabilidad social se relaciona con la posibilidad de comprender cómo y por qué diferentes grupos y sectores de la sociedad están sometidos de forma dinámica y heterogénea a procesos que atentan contra su subsistencia y capacidad de acceso a mayores niveles de bienestar. La complejidad de la noción de vulnerabilidad se expresa en diferentes niveles de análisis y en las dimensiones del desarrollo de las condiciones de vida social.

Combinando estas prerrogativas con nuestra experticia en operacionalizar la transdisciplina hemos implementado el Método VETAS $®$ en diversas instancias de educación e inclusión social tanto en instituciones privadas como públicas, desarrollando estrategias en distintos niveles de gestión y acción. 


\section{El método VETAS ${ }^{\circledR}$ como desarrollo de un método centrado en la Identidad}

Con base a la experiencia enriquecida en el quehacer diario y durante el proceso de creación y actualización continua como sistema abierto, fue necesario atravesar los ámbitos (social, educación, salud) en tres áreas y realizar una progresión en tres niveles distintos que implican un grado de complejidad creciente. En ella se van incluyendo en profundidad aspectos técnicos, de la persona del profesional y de su ámbito de influencia.

Las tres áreas identificadas son:

la persona

la técnica

el liderazgo

La persona: es el área relacionada con la actitud. La actitud implica el desarrollo personal en función del potencial emocional, intelectual y espiritual que está estrechamente vinculado con las cualidades innatas de cada individuo.

La técnica: es el área relacionada con la aptitud, la cual engloba todas las herramientas en relación a la profesión, experiencia y formación académica. Es el repertorio de conocimientos y técnicas aprendidas, de diferentes modalidades y que se aplican con pertinencia en la actividad diaria.

El liderazgo: es el área que expresa la excelencia y sirve como fundamento para desarrollar cambios e influir, gestionar y promover nuevos métodos para dar respuestas a la complejidad creciente

Las áreas anteriormente mencionadas se desarrollan en tres niveles identificados:

de la disciplina a la transdisciplina

la actitud y equipo transdisciplinario

la gestión y acción transdisciplinaria 


\section{Nivel de la disciplina a la transdisciplina}

Recoge el reto de la construcción activa de un lenguaje común.

Es el primer paso para lograr el lenguaje común que permita una comunicación plena.

El profesional se reconoce en su disciplina y comienza a considerar la necesidad de articular con otros miembros del equipo, que pueden representar o no, otras disciplinas y modos de aproximación a la investigación.

Los dispositivos hasta ahora implementados se han basado en intervenciones que intentan abordar los problemas complejos desde múltiples abordajes, pero con carencias en la integración.

Emerge así la necesidad de un enfoque que entrelace todas las miradas disciplinares y genere intervenciones que apunten a las múltiples necesidades y problemas reales.

El objetivo general del abordaje de este nivel es:

Adquirir un marco innovador en la articulación de conocimientos, que posibilite la centralidad de la identidad, a través del análisis de la complejidad y la acción transdisciplinaria.

Los objetivos específicos son:

Elaborar soluciones metodológicas en diagnósticos complejos en las áreas de salud, educación y acción social.

Actualizar conocimientos en conceptos inherentes a la práctica en el ámbito del ejercicio profesional.

Resignificar la importancia del proceso de evaluación desde la perspectiva transdisciplinaria acercándonos a una nueva perspectiva del conocer.

Desarrollar una actitud profesional basada en la articulación entre disciplinas, el profesionalismo, la integridad y el respeto por la diversidad. 
Los contenidos que se trabajan con el equipo son:

Transcausalidad, pensamiento complejo y la transdisciplinariedad.

Fundamentos para un Cambio de paradigma en salud, educación y acción social. Introducción a la metodología de la investigación en transdisciplinariedad.

\section{Nivel de la actitud transdisciplinaria y el trabajo en equipo. Del Yo al Nosotros}

En este nivel se desarrollan habilidades en pos de un objetivo común. El trabajo en equipo permite el intercambio de roles y funciones generando un clima laboral de bienestar y enriquecimiento mutuo. El profesional realiza su tarea pensando en términos de proceso y articula su saber generando estrategias con profesionales de otras áreas. Procura respuestas superadoras en el trabajo conjunto. Esta actitud según Nicolescu (2009) es "la capacidad individual o social de manteneruna orientación constante, inmutable, cualquiera sea la complejidad de una situación y losavatares de la vida" (p.65).Esta orientación debe atravesar los diferentes niveles de realidad y sus correspondientes niveles de percepción. Los objetivos de este nivel son:

Desarrollar una comunicación de excelencia en la legitimación del Otro que faciliteel compromiso hacia un lenguaje común.

Gestionar los procesos para la planificación estratégica en pos de orientarse a medir los resultados en tiempo y forma.

Lograr una sinergia entre los miembros del equipo, tornándolos más conscientes e integrando los objetivos organizacionales con los individuales.

Fomentar el respeto humano y profesional manejando conflictos comofactor de aprendizaje, estimulando el valor de la cooperación. 
Desempeñar su práctica con excelencia técnica y calidad humana.

Aprender a trabajar en equipo para potenciar la experiencia de la diversidad y la unidad en los profesionales.

Los contenidos abordados en este nivel son:

Formación en la actitud transdisciplinaria.

Identidad y misión personal.

Equipos transdisciplinarios.

La comunicación en equipos transdisciplinarios: del Yo al Nosotros.

Planificación estratégica y lenguaje común.

\section{Nivel de la gestión organizacional y la acción transdisciplinaria}

Recoge el reto de la gestión del cambio en nuestro ámbito de influencia.

Una organización saludable es aquella que estimula y desarrolla la misión personal de cada uno de sus integrantes, que se manifiesta a través de sus habilidades y talentos, promoviendo el valor de la salud como la base de una actitud laboral efectiva, posibilitando así el encuentro con la misión de la organización para la cual se trabaja, potenciando el nivel de identidad y pertenencia a la misma.

En este nivel nos proponemos liderar el cambio en la organización a través de la gestión transdisciplinaria. Nuestra intención es desarrollar desde esta metodología transdisciplinaria, la visión del funcionamiento de las organizaciones. Este nivel se enfoca en aportar innovación, fijar el rumbo de la organización, definir la cultura, motivar e inspirar y armonizar a las personas.

La metodología transdisciplinaria implica un trabajo en procesos continuos de desarrollo sistémico, elaborados sobre la base de entender las organizaciones como un producto centrado en las personas que la componen. En este nivel se busca que el rol sea expresión de la misión 
personal, desarrollando capacidades y talentos que multiplicarán las alternativas para responder a las presiones que plantea la complejidad en las organizaciones de neurorehabilitación. Los objetivos de este nivel son:

Alcanzar la calidad de los servicios basados en la identidad de las personas.

Desarrollar habilidades para la gestión de objetivos organizacionales.

Desarrollar competencias de liderazgo, articulando la gestión organizacionaly el trabajo en equipos transdisciplinarios.

Concientizar el nivel de identidad expresado en el rol que ejerce cada profesional en la organización.

Incorporar herramientas de planificación y pensamiento estratégico para agregar valor en su área de influencia.

Colaborar con una mejora en el estilo de afrontamiento de las situaciones generadoras de estrés del ejercicio profesional, en el marco del actuar en un contexto de complejidad creciente.

\section{CONCLUSIONES}

La elaboración de metodologías transdisciplinarias para la investigación-transformación en diversos ámbitos es una necesidad que requiere ser atendida mediante trabajo colaborativo y atención a la diversidad de situaciones sociales complejas.

El Método VETAS® tiene en común con otras metodologías transdisciplinarias los ideales epistemológicos comunes a las teorías de la complejidad, el pensamiento complejo, y el basamento que ofrece el Manifiesto publicado por Nicolescu en 1996. Esa comunidad en los presupuestos de ruptura con la racionalidad clásica se acompaña de diferenciadores específicos. Entre ellos sobresalen el examen del contexto, la acumulación de experiencia práctica en actividades de investigación, atención de salud, trabajo social y educación, la 
centralidad de la identidad en la construcción metodológica y la permanente apertura al diálogo con los diversos componentes del contexto, representados por los actores, los saberes y las problemáticas.

El Método VETAS®, constituido en base al pensamiento complejo y la transdisciplinariedad puede ser aplicable a otras áreas y organizaciones. Deberá evitarse cualquier intento de traslación como si se tratara de una receta operativa. Por el contrario, se requiere la construcción continua con base en el contexto, los actores, saberes y procesos que convergen en él. Solo en el respeto y la comprensión de cada identidad expresada y reconocida como una unidad compleja es que resulta pertinente la aplicación del método.

Incorporar métodos como el desarrollado, implica conciencia del cambio de paradigma que se encuentra gestando y compromiso con un acercamiento y comprensión de la realidad que supere la primacía de saberes por defecto, así como prácticas más humanizadas que pongan en valor el desarrollo de las personas y la construcción de conocimientos pertinentes.

Experiencias convergentes se han desarrollado en el terreno educativo. Además de los trabajos desarrollados por el Instituto de Docencia e Investigación de Fundación AlunCo, y los reunidos en varios textos (Delgado, 2019, Mussallam 2019). En el desarrollo actual de metodologías vinculadas al Método VETAS®, sobresale la experiencia desarrollada entre 2007 y 2016 en Italia bajo la dirección de G. Finocchiaro (2009, 2016), que condujo al desarrollo del método EDUCANDO.IT. Por su orientación transdisciplinaria y la convergencia en la centralidad de la identidad se desarrolla en la actualidad en el Método VETAS® EDUCANDO.IT que requiere por su especificidad ser explicado en otras publicaciones. 


\section{REFERENCIAS BIBLIOGRÁFICAS}

Delgado, C. (2012). Conocimiento, conocimientos, diálogo de saberes. En Valdés, F., y León, Y. (2012). La filosofía en su tiempo histórico. La Habana: Ciencias Sociales, Ruth Cuadernos de Pensamiento Crítico, No. 0, 2012, pp. 159-180).

Delgado, C. (2018). Transdisciplinariedad. En Mussallam, M. B. (ed.) (2018). Guia Global de Ética, Principios, Políticas y Prácticas en Educación Equilibrada e Inclusiva. (pp. 35-53) Ginebra, Suiza: ERF.

Delgado, C (2019). Investigar desde el pensamiento complejo. Mexico: Multiversidad Mundo Real Edgar Morin.

Finocchiaro, G. (2009). Autonomia e innovazione: Curricolo a 20 ore. Palermo: Carlo Saladino.

Finocchiaro, G (2016). La scuola di chi: Come realizzare la centralità degli alunni. Palermo: Carlo Saladino.

Freire, P. (1969). Pedagogía del oprimido. Disponible en: http://www.servicioskoinonia.org/biblioteca/general/FreirePedagogiadelOprimido.pdf, s.1,s.a.

Hurtado, C. (2015). La transformación educativa. ¿Para qué y por qué educar?, Buenos Aires: La Colmena.

Klein, J. T. (2013). The transdisciplinary Momentum, Integral Review, special issue International Symposium: Research Across Boundaries, Part 1, vol. 9, no. 2, 2013, pp. 189-199, http://www.integral-review.org/ issues/vol_9_no_2_jun_2013_full_issue.pdf\#page=194.

Lyotard, J. F. (1981). La condizione postmoderna: Rapporto sul sapere. Milano: Feltrinelli. Luengo, E. (2015). De la insatisfacción metodológica al encuentro con la transdisciplina. En Gómez-Gómez, E. N. y Arboleda-Gómez, R. (coords.), Diálogos sobre la 
transdisciplina. Los investigadores y sus objetos de estudio, Guadalajara, ITESO, 2015, http://hdl.handle.net/11117/3058.

Morin, E. (1999). Los siete saberes necesarios para la educación del futuro. París, Francia: UNESCO.

Morin, E. (2000). Antropología de la libertad. Gazeta de Antropología, no. 16, Texto 16-01 Disponible en: http://www.ugr.es/\%7Epwlac/G16_01Edgar_Morin.html

Morin, E. (2012). La via: Per l'avvenire dell'umanità. Milano: Raffaello Cortina.

Morin, E. (2015). Insegnare a vivere: Manifesto per l'educazione. Milano: Raffaello Cortina.

Morin, E. (2016). Sette lezioni sul pensiero globale. Milano: Raffaello Cortina.

Mussallam, M. B. (ed.) (2018). Global Guide of Ethics, Principles, Policies, and Practices in Balanced and Inclusive Education. Ginebra, Suiza: ERF.

Nicolescu, B. (1996) La transdisciplinariedad. Manifiesto. Mónaco: Du Rocher.

Nicolescu, B. (2005). Transdisciplinarity - Past, present and future. Palestra apresentada no II Congresso Mundial de Transdisciplinaridade 06 a 12 de setembro de 2005 Vila Velha/Vitória - SC - Brasil pp. 1-24. Disponible en http://cetrans.com.br/assets/textos/multidisciplinario-past-present-and-future.pdf

Nicolescu, B. (2009). La transdisciplinariedad. Manifiesto. México: Multiversidad Mundo Real Edgar Morin.

Nicolescu, B., Yeh, R. T., Ertas, A. (eds.) (2019). Being Transdisciplinary. USA: ATLAS Publishing.

UNESCO (2015). Replantear la educación. ¿Hacia un bien común mundial? París, Francia: UNESCO. 